\title{
Magnetic resonance techniques for real-time assessment of thermal treatment
}

\section{Thaddeus Samulski}

Thaddeus V. Samulski, "Magnetic resonance techniques for real-time assessment of thermal treatment," Proc. SPIE 10297, Matching the Energy Source to the Clinical Need: A Critical Review, 102970G (24 January 2000); doi: $10.1117 / 12.375217$

SPIE. Event: Digital Optical Computing, 1990, Los Angeles, United States 


\title{
Magnetic Resonance Techniques for Real-Time Assessment of Thermal Treatment
}

\author{
Thaddeus V. Samulski, Ph.D. \\ Dept. of Radiation Oncology, Duke University Medical Center, \\ Durham, NC
}

\begin{abstract}
This paper reviews the application of MRI as a non-invasive technique for accessing temperature in thermal therapies. Two MR parameters, T1 relaxation and proton resonance frequency (PRF), have shown temperature sensitivity that is measurable with MRI. A third temperature dependent parameter associated with the self-diffusion of water, referred to as the apparent diffusion coefficient (ADC), is not intrinsic to nuclear magnetic resonance (NMR), but can be measured using MRI. These three parameters have been useful for quantitatively mapping temperature distributions in vivo. Each requires a tailored imaging technique. Each has pros and cons with regard to a given application. At this point in the development of MR image guided temperature mapping, the PRF shift technique is preferable, particularly with regard to non-invasive thermal ablation procedures. This approach has problems with stability and motion in procedures that require heating for extended time periods. Further development of MRI thermometry is required for long duration procedures.
\end{abstract}

\section{INTRODUCTION}

The use of magnetic resonance imaging (MRI) in thermal therapy has rapidly grown in the past decade and a half. The utilization is quite broad in scope including reports of using MRI for: tissue damage assessment $t^{1-7}$; blood flow and perfusion measurements ${ }^{8-10}$; and therapy prognoses and outcome ${ }^{11-13}$. I mention these applications not as an exhaustive review, but to give some measure of the use of MRI in thermal therapy other than the application for non-invasive real-time thermometry. This paper will focus on progress in thermometry techniques.

Since MRI is a relatively new imaging modality there may be readers who have little or no exposure to nuclear magnetic resonance (NMR) and its application in imaging. Therefore, this review is divided into three major sections. The first concerns the basic physics of NMR. The second is related to using NMR for non-invasive imaging. The third concerns the thermally sensitive imaging techniques that have been developed and tried in ex vivo and/or in vivo applications. Initial efforts to use MRI for thermal therapy began in the early 1980s. There has been continued interest and development in this idea ever since. This review of that progress is clearly positive and points to accelerated development in the future. 


\subsection{Nuclear Magnetic Resonance (NMR)}

Although NMR is fundamentally a quantum mechanical phenomenon, it is often intuitively described using a mixture of quantum and classical mechanics. This mixed model can be used to describe proton NMR that is primarily involved in magnetic resonance imaging of tissues. If a macroscopic sample of water is placed in a static external magnetic field, it becomes "magnetized". This magnetization does not happen instantaneously. It requires some characteristic time $T_{1}$ to reach an equilibriummagnetized state. The sources of this magnetization are the magnetic moments of hydrogen protons in the water molecules. This magnetic moment is a fundamental property of the proton. The origin of the proton magnetic moment $(\mu)$ can be classically modeled as a current loop with a magnitude equal to the current times the area of the loop (see Fig. 1).

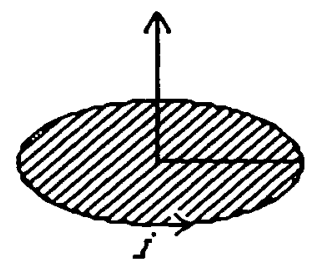

$$
\bar{\mu}=i \cdot \pi r^{2} \quad e q 1
$$

This magnetic moment can be easily related to the angular momentum of the loop by considering a mass, $m_{p}$, with charge, $\mathrm{e}^{+}$, moving with angular momentum, $m_{p} v r$. Thus,

$$
\bar{\mu}=\frac{e \bar{l}}{2 m_{p}}
$$

where $\bar{l}$ is the angular momentum, and the direction of $\bar{\mu}$ is the same as $\bar{l}$. Next we interject the quantum angular momentum for which we know the magnitude is $l=\hbar \sqrt{I(I+1)}$; where $\hbar$ is Planck's constant divided by $2 \pi$, and $I$ can only have integer or half integer values. For the proton $I=\frac{1}{2}$, therefore the magnitude of $l=\frac{\sqrt{3}}{2} \hbar$. In addition, quantum mechanics tells us that the direction of the quantum angular momentum cannot be known; we can only know the projection of the angular momentum along a given axis. In the case of the proton the projections are $\pm \frac{\hbar}{2}$. Also, since there are two possible projections for $\bar{\mu}$ along any given direction, there are two energy states for protons in an external magnetic field. 
Needless to say, when we use the known value for proton mass, charge, and angular momentum, the predicted magnitude of the magnetic moment does not agree with measurement. So a correction factor is assigned:

$$
\bar{\mu}=g \frac{e}{2 m_{p}} \bar{l} \quad \text { eq } 3 \mathrm{a}
$$

where the g-factor is scaled such that $\mu$ agrees with measured values.

This relationship is also written:

$$
\bar{\mu}=\bar{\gamma}
$$

with $\gamma$ being defined as the gyromagnetic ratio, equal to $2.675 \times 10^{8} \mathrm{~Hz}$ per Tesla for protons.

The interaction of the proton magnetic moment with an external magnetic field, $\bar{B}$, has energy given by:

$$
U=-\bar{\mu} \cdot \bar{B}
$$

eq $4 a$

and a torque given by:

$$
\bar{\tau}=\bar{\mu} \times \bar{B}
$$

The first equation (4a) implies a lower energy state exists when the magnetic moment projection is aligned with the direction of the external magnetic field. The second equation implies that magnetic moment will precess around the direction defined by the magnetic field with angular frequency $\omega_{o}=\gamma B$. This is known as the "Larmor frequency", which is in the radio frequency (RF) range for imaging applications.

As a two energy state system in thermal equilibrium, the ratio of magnetic moments aligned with the external B-field to moments aligned opposite to the B-field will be less than one from the

$$
\frac{N \downarrow}{N \uparrow}=e^{-2 \mu B / k T}
$$

The bulk magnetization, $\bar{M}=\sum_{i} \bar{\mu}_{i}$ over all protons in a unit volume. Therefore in thermal equilibrium:

$$
M=N \mu \tanh (\mu B / k T)
$$

eq 6

where $\mathrm{N}$ is the number of proton per unit volume. 
The equation of motion for $\bar{M}$ in a static field $\bar{B}_{o}$ is given by:

$$
\frac{d \bar{M}}{d t}=\gamma \bar{M} \times \bar{B}_{o}
$$

eq 7

which describes a precession of $\bar{M}$ around the direction of $\bar{B}_{o}$ with an angular frequency of $\omega_{o}=\gamma B$. However, in the equilibrium state, $\bar{M}$ will always be aligned with $\bar{B}_{o}$. For the non-equilibrium case the system can be described with three equations that include transient terms that drive the magnetization to the equilibrium-state. These are referred to as the Bloch equations:

$$
\begin{array}{ll}
\frac{d M_{z}}{d t}=\gamma\left(\bar{M} \times \bar{B}_{o}\right)_{z}+\frac{M_{o}-M_{z}}{T_{1}} & \text { eq 8a } \\
\frac{d M_{x}}{d t}=\gamma\left(\bar{M} \times \bar{B}_{o}\right)_{x}-\frac{M_{x}}{T_{2}} & \text { eq 8b } \\
\frac{d M_{y}}{d t}=\gamma\left(\bar{M} \times \bar{B}_{o}\right)_{y}-\frac{M_{y}}{T_{2}} & \text { eq } 8 \mathrm{c}
\end{array}
$$

where $\bar{B}_{o}$ is chosen to be along the z-axis $\bar{B}_{o}=B_{o} \hat{z}$. These equations have solutions of the form:

$$
M_{z}(t)=M_{o}\left(1-e^{-\frac{t}{T_{1}}}\right) \quad \text { eq } 9 \mathrm{a}
$$

which for eq. $9 \mathrm{a}$ implies that $M_{z}=M_{o}$ as $t \rightarrow \infty$.

Solutions for eqs $9 \mathrm{~b}, 9 \mathrm{c}$ are of the form:

$$
\begin{aligned}
& M_{x}=M_{x y} \cos \left(\omega_{o} t\right) e^{-\frac{t}{T_{2}}} \quad \text { eq } 9 \mathrm{~b} \\
& M_{y}=-M_{x y} \sin \left(\omega_{o} t\right) e^{-\frac{t}{T_{2}}} \quad \text { eq } 9 \mathrm{c}
\end{aligned}
$$

The transverse magnetization, $M_{x y} \rightarrow 0$ as $t \rightarrow \infty$.

The characteristic times $T_{1}$ and $T_{2}$ are referred to as the spin-lattice relaxation time constant and the spin-spin relaxation time constant, respectively. The $T_{1}$ relaxation involves both conservation of energy and conservation of angular momentum. Therefore, this relaxation has to exchange energy $h \omega_{o}$ and angular momentum $\frac{\hbar}{2}$ with the surrounding medium or lattice. Consequently we expect $T_{1}$ to have temperature dependence proportional to $e^{-\varepsilon_{a} / k T}$. The $T_{2}$ relaxation does not require any energy 
exchange since there is no magnetic field along the $\mathrm{x}$ or $\mathrm{y}$ axis. Thus, it only requires conservation of angular momentum in units of $\frac{\hbar}{2}$. This interaction involves fluctuations in the local magnetic field seen by the proton. Such fluctuations are often associated with unpaired magnetic moments such as that found in paramagnetic materials. These fluctuations are temperature dependent as well: $\propto s \eta e^{-\varepsilon / k T}$ where $s$ is a geometric shape factor and $\eta$ is the local viscosity. For tissues $T_{1}$ at 1.5 Tesla ranges from $\sim 0.2-0.9$ sec and $T_{2}$ range from $40-100 \mathrm{msec}$. Since the magnitude of $T_{2}$ is an order of magnitude less than $T_{1}$, temperature changes can be more precisely measured using the $T_{1}$ relaxation.

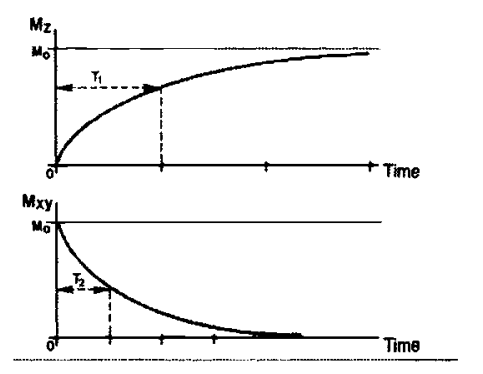

Fig. 2: The transient behavior of the longitudinal- $\mathrm{z}$ and transverse $\mathrm{x}, \mathrm{y}$ magnetizations are independently governed by $T_{1}$ and $T_{2}$

Up to this point we have been concerned with bulk magnetization associated with a static magnetic field and there is no resonance phenomenon. Resonance occurs when we combine a time varying magnetic field in the transverse plane with respect to the static magnetic field. In this case we have:

$$
\bar{B}=\left[B_{l} \cos (\omega t)_{\hat{x}}-B_{l} \sin (\omega t)_{\hat{y}} B_{o \hat{z}}\right] \quad \text { eq } 10
$$

The $\bar{B}_{1}$ field is rotating in the $\mathrm{x}, \mathrm{y}$ plane at frequency $\omega$, and is assumed to be much smaller than $B_{o} \hat{z}\left(\bar{B}_{l} x y \ll B_{o} \hat{z}\right)$. Using this total field in the Bloch equations yields general solutions of the form:

$$
\begin{array}{r}
M_{x}=M_{x y} \cos (\omega t+\phi) \cdot e^{-\frac{t}{T_{z}}} \\
M_{y}=M_{x y} \sin (\omega t+\phi) \cdot e^{-\frac{t}{T_{2}}} \\
M_{z} \cong M_{o}\left(1-e^{-\frac{t}{T_{1}}}\right)
\end{array}
$$

Note $M_{z} \cong M_{o}$ since $B_{o}>B_{1}$. The angle, $\phi$, is the phase difference between $\bar{B}_{l}$ and $\bar{M}$.

Upon substitution into the Bloch equations, we can solve for $M_{x y}$, the amplitude of the magnetization in the $x-y$ plane. 


$$
M_{x y}=\frac{\gamma M_{o} T_{2} B_{1}}{\left[1+\left(\omega_{o}-\omega\right)^{2} T_{2}^{2}\right]^{\frac{1}{2}}}
$$

This result has a resonance behavior as $\omega \rightarrow \omega_{o}$. The amplitude of the transverse magnetization grows as $\omega$ approaches the resonance frequency $\omega_{o}$. Also, note that $M_{x y}$ is proportional to $M_{o}$.

The energy absorption rate of the system is:

$$
P=\bar{B} \cdot \frac{d \bar{M}}{d t}
$$

and open substitution:

$$
P=\frac{\omega \gamma M_{o} T_{2} B_{l}^{2}}{1+\left(\omega_{o}-\omega\right)^{2} T_{2}^{2}} \quad \text { eq } 13 b
$$

Therefore there is resonant absorption as well.

In this semi-classical model, the magnetization $M_{o}$ is initially aligned with the static magnetic field $B_{o} \hat{z}$. The application of a weak, $\left(B_{l}(\omega)_{\hat{x} \hat{y}} \ll B_{o} \hat{z}\right)$, rotating magnetic field in the xy plane exerts a torque $\bar{M}_{o} \times \bar{B}_{l}(\omega) \hat{x} \hat{y}$. However, for any $\omega$ other than $\omega_{o}$ the torque will average to zero. When $\omega=\omega_{o}$ the angular relationship between $\bar{M}_{o}$ and $\bar{B}_{1}\left(\omega_{o}\right) \hat{x} \hat{y}$ remains fixed since $\bar{M}_{o}$ also presseses at frequency $\omega_{o}$. Therefore, $M_{o} \times\left[B_{o} \hat{z}+\bar{B}_{l}\left(\omega_{o}\right) x y\right]$ will induce a precession about both magnetic fields. The resulting motion in the laboratory frame of reference describes $\bar{M}_{o}$ as spiraling down away from $+\hat{z}$ towards $-\hat{z}$ (see Fig, 3 ).

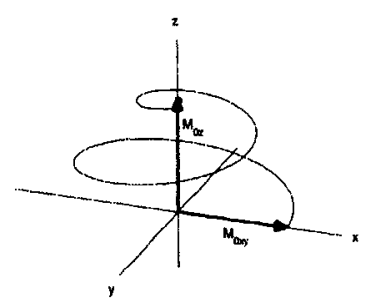

Fig.3: The rotating $B_{1}$ field tips the magnetization away from the $\mathrm{z}$-axis

Depending on the magnitude and time duration of $B_{1}\left(\omega_{o}\right) \hat{x} \hat{y}, \bar{M}_{o}$ can be tilted to any polar angle $\theta$ between 0 and $180^{\circ}$. Once $\bar{M}_{o}$ has a component in the transverse plane it can be detected via magnetic induction using a loop antenna properly oriented relative to the rotating $\bar{M}_{o} x y$ component. The maximum induced current will occur when

$\bar{B}_{1}\left(\omega_{o}\right)$ rotates $\bar{M}_{o} 90^{\circ}$ relative to $B_{o} \hat{z}$. Once $\bar{M}_{o}$ is oriented at some angle away 
from $B_{o} \hat{z}, \bar{M}_{o} x y$ will decay as $e^{-\frac{t}{T_{2}}}$. The induction signal will have the form of $M_{o} x y \cos \left(\omega_{o} t\right) e^{-\frac{t}{T_{2}^{*}}}$. This signal decreases with a time constant $T_{2}^{*}<T_{2}$. This signal is referred to as the free induction decay (FID) (Fig. 4).

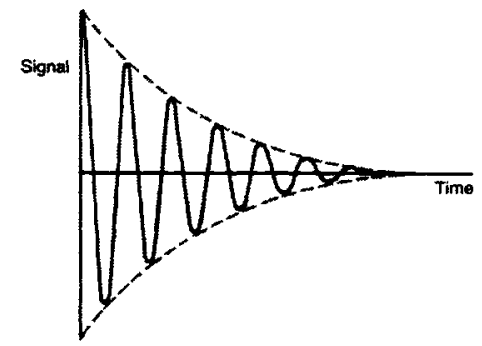

Fig.4: The rotating magnetization in the $\mathrm{x}$ y plane induces an emf signal with frequency $\omega_{o}$ with a decay time: $T_{2}^{*}$

The free induction signal can be associated with the coherent precession of the projection of proton magnetic moments in the transverse plane. That is, all protons are forced by the $B_{1}\left(\omega_{o}\right)$ field to precess in phase. However, this coherent precession can not be sustained. Local field variations (i.e. the field at the location of a given proton) cause the coherent precession to dephase (see Fig. 5). The time constant $T_{2}^{*}$ is a measure of the average time that the proton spins precesses in phase.
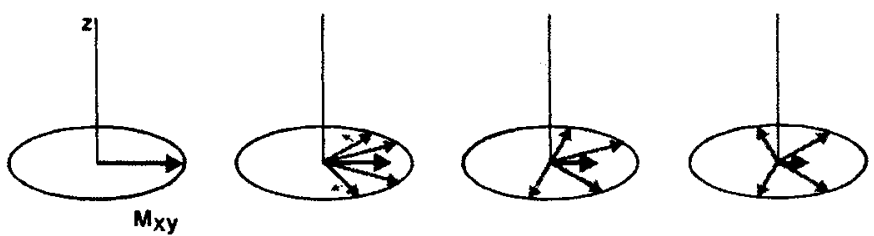

Fig.5

Because the dephasing is associated with random and non-random variations of the local field, an interesting rephasing echo phenomenon can be observed in the induction signal. A second $B_{1}\left(\omega_{o}\right)$ pulse of appropriate magnitude and duration can be used to flip the transverse plane of magnetization by $180^{\circ}$. This flip will cause the reversal of the dephasing associated with the non-random variations in the local field and produce an echo signal. This can be understood if one observes the dephasing of the $M_{\sigma} x y$ in the frame of reference rotating at frequency $\omega_{o}$. In this view, protons in a relatively weaker field $\left(\omega<\omega_{o}\right)$ will appear to precess counter clockwise relative to precession at $\omega_{o}$. Protons located in relatively higher local fields will appear to precess clockwise with $\left(\omega>\omega_{o}\right)$, thereby causing the vector sum of spin projection to decrease (Fig 5). The $180^{\circ} B_{1 x, y}$ pulse has the effect of reversing the direction of precession thereby causing the $M_{o x y}$ magnetization to refocus and produce the echo signal (Fig.6). 


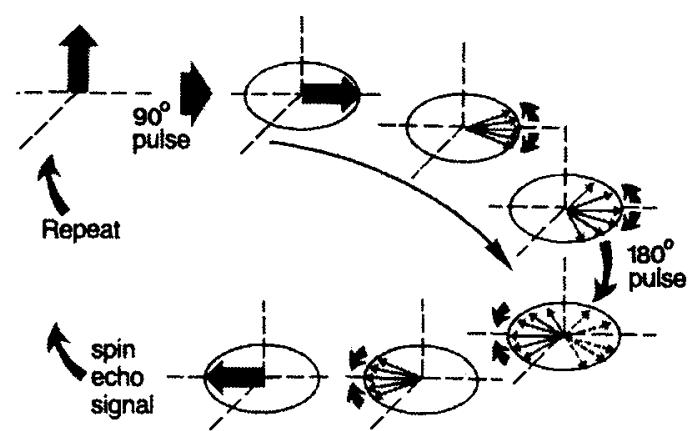

Fig.6: Schematic of the spin echo technique

Using a series of $180^{\circ}$ pulses, a train of successive echoes can be produced. The magnitude of the echo signals decrease with the time constant $T_{2}$. This technique is commonly used in NMR.

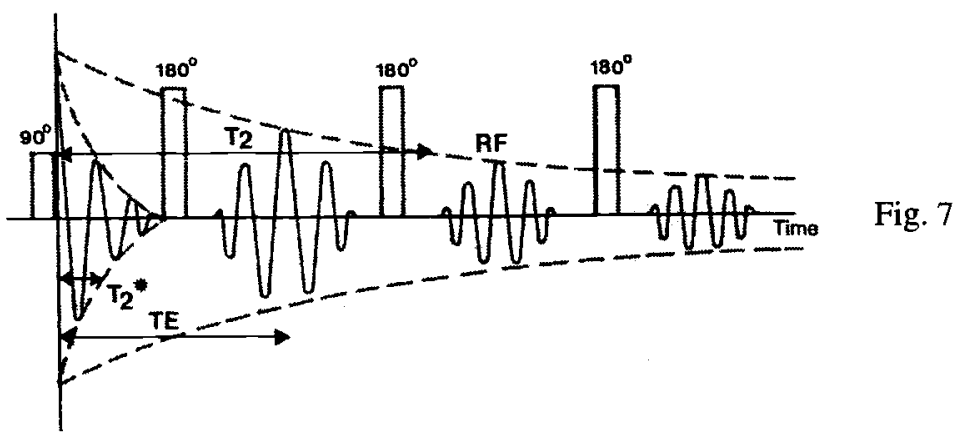

\subsection{Magnetic Resonance Imaging}

Adapting NMR to imaging requires the resonance excitation of a sub-volume of the object to be imaged and measuring the FID or spin echo signal. The signal emitted by the sub-volume will depend on the proton density and the relaxation times $T_{1}$ and $T_{2}$. The local excitation can be accomplished by imposing a known spatial variation in the magnetization field $B_{o \bar{z}}$. This variation can be achieved by adding smaller magnetic fields along the $z$-axis that vary in magnitude as a function of position $(x, y, z)$ in the image volume. These fields usually vary linearly with position and are referred to as gradient fields. With such fields superimposed on the $B_{o \bar{z}}$ field each position in space will effectively have a different resonance frequency. In principle, one could selectively excite each voxel with the appropriate resonance frequency, measures the resultant signal, and assemble the spin density image. However, this approach is not practical with regard to the data acquisition time required. An alternative technique using time and spatially dependent gradient fields is much more efficient. A commonly used technique is illustrated in Fig. 8 


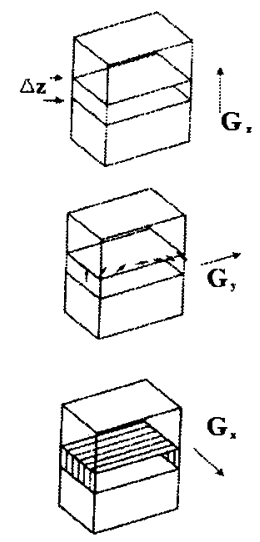

Fig. 8: Three gradient fields are applied at different times and in different directions to: excite a slice along $z$, phase encode in $y$, and frequency encode in $x$

Here a 2-D slice of thickness $\Delta \mathrm{z}$ is excited to resonance by applying a $90^{\circ} \mathrm{RF}$ pulse in the presence of the $\mathbf{z}$-gradient field. The magnitude of the $\mathbf{z}$ gradient and the RF pulse-shape and bandwidth determine the slice thickness. Prior to decting the FID or spin echo signal, a y-gradient is applied for a time $t_{y}$. This gradient advances the spin precession in proportion to the strength and time duration it has been applied. This is referred to as phase encoding of the signal. The FID or spin echo signal is acquired in the presence of the $\mathrm{x}$-gradient. This encodes the signal frequency in the $\mathrm{x}$-direction. If we consider the magnetization in the $\mathrm{z}$-slice of the object, we can write an expression for the FID or echo signal:

$S(t)_{z}=e^{-t / T_{2}} \iint m(x, y)_{z} \exp (-i \gamma)\left[B_{o}+\int_{o}^{t} G y(\tau) \cdot y d \tau+\int_{o}^{t} G x(\tau) \cdot x d \tau\right] d x d y \quad$ eq 14

where $S(t)_{z}$ is the signal from the precessing magnetization $m(x, y)_{z}$ in the slice location $\mathbf{z}$.

We can define:

$$
\begin{aligned}
& k_{x}(t)=\frac{\gamma}{2 \pi} \int_{o}^{t} G_{x}(\tau) d \tau \\
& k_{y}(t)=\frac{\gamma}{2 \pi} \int_{o}^{t} G_{y}(\tau) d \tau
\end{aligned}
$$

to give:

$$
S\left(k_{x}(t), k_{y}(t)\right)_{z}=e^{-\frac{t}{T_{2}}} e^{-i \omega_{o} t} \iint m(x, y)_{z} e^{-i 2 \pi\left(k_{x}(t) x+k_{y}(t) y\right)} d_{x} d_{y} \quad \text { eq } 16
$$

We can easily identify that the double integral over $\mathrm{x}$ and $\mathrm{y}$ is the 2-D Fourier transform of $m(x, y)$ at time $=\mathrm{t}$. Therefore, the inverse transform of the function $S\left(k_{x}(t), k_{y}(t)\right)$ in 
the $k_{x}, k_{y}$ plane will produce the proton magnetization density, $m(x, y)_{z}$. In order to do this inversion we have to know the value of $S\left(k_{x}(t), k_{y}(t)\right)$ in the $k_{x} k_{y}$ plane. This can be determined by incrementing the time variable in $k_{x}(t), k_{y}(t)$ and collecting the respective signals. This process of repeating the signal measurement with different time weighted gradients continues until we have enough $S\left(k_{x}(t), k_{y}(t)\right)$ data to numerically carry out the inverse Fourier transform. There are several ways of varying the gradient fields for gathering the k-space data. One most commonly used technique increments $G_{y}(t)$ in predefined time steps $t_{n}$ prior to the signal measurement. Subsequently, $G_{x}(t)$ is applied during the signal measurement. This method of encoding is illustrated in Fig. 9.

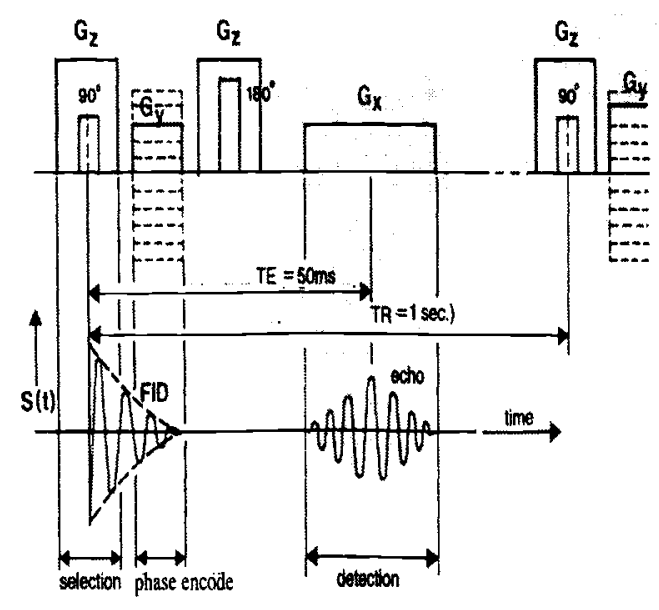

Fig. 9: The pulse diagram for image acquisition using the spin echo technique. The pulse sequence is repeated at time, TR, with different Gy

The factor $e^{-\omega_{o} t}$ in equation 16 carries no information relevant to $m(x, y)$ and is removed by demodulation. The factor $e^{-\frac{t}{T_{2}}}$ is a function of $\mathrm{x}, \mathrm{y}$ and can be incorporated into $m(x, y)$. Thus, the demodulated signal is:

$$
S\left(t, t_{y}\right)_{z}=\iint m(x, y)_{z} \cdot \exp \left[-i \gamma\left(G_{y} y \cdot t_{y}+G_{x} x \cdot t\right)\right] d y d x \quad \text { eq } 17
$$

The signal we record has the form:

$$
S_{r}(t)=A(t) \cos \left(\omega_{o} t+\omega_{m}(t)\right)
$$

where $A(t)$ is the amplitude and $\omega_{m}(t)$ is the gradient modulated frequency. This signal is split and mixed (multiplied) with $\cos \left(\omega_{o} t\right)$ and $\sin \left(\omega_{o}(t)\right.$ signals. The mixed signal is the sum of two components: one oscillating at $\left(2 \omega_{o}+\omega_{m}(t)\right)$ and the other at $\omega_{m}(t)$. After mixing, the quadrature signals are filtered to remove the high 
$\left(2 \omega_{o}+\omega_{m}(t)\right)$ frequency component. The net result is two signals that are $90^{\circ}$ out phase:

$$
\frac{A(t)}{2} \cos \left(\omega_{m}(t)\right) \text { and } \frac{A(t)}{2} \sin \left(\omega_{m}(t)\right)
$$

These are referred to as the real and imaginary signals. The are independently reconstructed to form two images, also referred to as the real and imaginary images. As complex numbers these image data are displayed as amplitude, $m(x, y)$ and phase, $\phi(x, y)$, images, with the amplitude image representing the imaged object:

\subsection{Contrast in MRI}

The MR image has intensity depending on the proton density and the $T_{1}, T_{2}$ relaxation time constants. The difference of these parameters in tissues is the source of image contrast. Also, the image acquisition technique can be manipulated to optimize the contrast. Figures $(10 \mathrm{a}, 10 \mathrm{~b})$ illustrate how the timing in the acquisition pulse sequence can optimize the contrast for tissues that have different $T_{1}, T_{2}$ values.

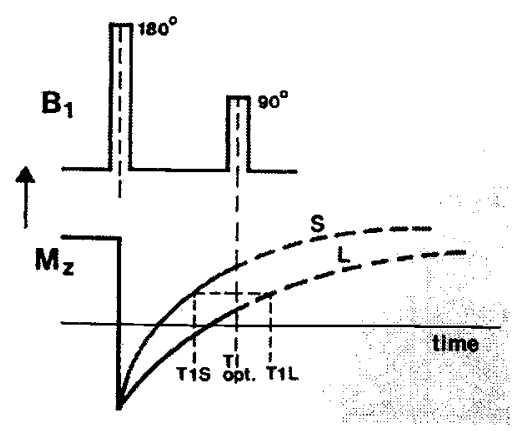

Fig. 10a

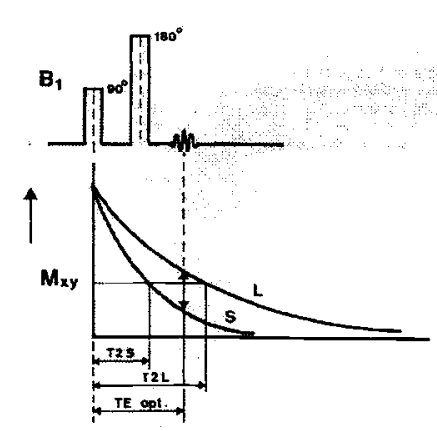

Fig. 10b

The ability to manipulate image contrast in MRI is far superior to other imaging modalities. Contrast agents are also available for MRI. These agents often utilize paramagnet materials or nuclei with large magnetic moments that shorten the relaxation times to manipulate the MR signal contrast.

\subsection{Temperature dependence in MRI}

In the NMR review we noted that the bulk magnetization, $M_{o,}$ and relaxation times $T_{1}, T_{2}$, were temperature dependent via the Boltzmann relation, $e^{-\frac{\varepsilon_{a}}{k T}}$, where $\varepsilon_{a}$ is the activation 
energy governing the parameter over some temperature range. In the case of the bulk magnetization, $M_{o}$, the activation energy is $\hbar \omega_{o}$. For a magnet of 1.5 Tesla $\hbar \omega_{o}<k T$ and $\frac{d M_{o}}{d T} \approx 10^{-7} \frac{\text { Joule }}{{ }^{\circ} K m^{3} T e s l a}$. Therefore one would need an extremely precise measurement technique to use this parameter to detect temperature from the MR signal. Of these three MR parameters, $T_{1}$ is the best candidate. In 1980, Lewa and Majewska ${ }^{14}$ measured $T_{1}$ temperature dependence animal and human tissues (liver, spleen, heart, lung, and tumor) using a NMR spectrometer. They reported $\varepsilon_{a}$ values of $0.06 \mathrm{eV}$ to $0.09 \mathrm{eV}$ for the temperature range of $10-40^{\circ} \mathrm{C}$. This implies: $\frac{d T_{1}}{d T} \approx 0.01 \frac{\mathrm{sec}}{{ }^{\circ} \mathrm{K}}$ (i.e. about a $1 \%$ change in $T_{1}$ per ${ }^{\circ} \mathrm{C}$ ). They also found that $T_{1}$ had an irreversible temperature dependence if the samples were heated above a maximum temperature of $40^{\circ} \mathrm{C}$. Above this threshold, the activation energies changed by factors of 2 to 3 , depending on the tissue type. They associated this effect with protein denaturation.

The feasibility of using MRI for measuring temperature changes in water and blood was reported by Parker ${ }^{15}$. This work demonstrated linear relationship between $T_{1}$ and temperature over the range of 1 to $48^{\circ} \mathrm{C}$ in water. The achieved temperature resolution was on the order of $1-2^{\circ} \mathrm{C}$. The data from blood suggest a change in activation energy at higher temperature as noted above.

Temperature measurements in vivo using $T_{1}$ changes were reported by Dickinson et.al. ${ }^{16}$. A $27 \mathrm{MHz}$ RF source was used for tissue heating. Parallel plate electrodes were used on rabbit thigh muscle and on the calf of one human volunteer. A simplified version of the saturation recovery sequence was used for MR imaging. The heating RF pulse was gated such that it was off during the image acquisition. A linear dependence on $T_{1}$ was observed in these experiments. Temperature resolution was on the order of $1-2^{\circ} \mathrm{C}$. In retrospect, this effort marked the beginning of a period of accelerated use of MRI for visualizing thermal energy distributions and thermal damage tissues.

The combined use of laser thermal ablation and MR $T_{1}$ and $T_{2}$ weighted images was reported by Jolesz et.al. ${ }^{2}$. They reconfirmed the nonlinear response between temperature and these MR parameters. Furthermore, their visualization of the thermal distribution using MR was extremely useful for this invasive application. Similar results for ex vivo and in vivo tissues are reported for the combination of MRI with thermal ablation and cryo-ablation ${ }^{3}$. Hynynen et. al. ${ }^{4}$ applied this use of $T_{1}, T_{2}$ contrast to non-invasive thermal ablation using high- intensity focused ultrasound. This work demonstrated that a non-invasive energy source could be compatibly used in the MR scanner. It also showed that thermal ablation could visualized in vivo using $T_{1}$ or $T_{2}$-weighted images, the latter being preferable.

A study for optimizing $T_{1}$ temperature contrast was made by Cline et.al ${ }^{17}$. This tested the feasibility of using a fast gradient echo sequence to capture the temperature distribution in tissue associated with a high-intensity (US) energy pulse. This work was done in ex vivo bovine muscle at temperatures below $40^{\circ} \mathrm{C}$. The $T_{1}$ temperature sensitivity was found to be $-1.4 \%$ per ${ }^{\circ} \mathrm{C}$ in this range. This temperature sensitivity was used to establish an optimized contrast vs. temperature relation. This in turn was used in with fast gradient echo imaging technique ( $\mathrm{TE}=3.4 \mathrm{msec}$., $\mathrm{TR}=10.6 \mathrm{msec}$.) to measure the temperature 
distribution in the US focus. This demonstration of using $T_{I}$-thermometry to both visually identify and quantify a temperature distribution was an important step in the development of image guided MR thermal ablation.

The use of $T_{1}, T_{2}$ MR weighted images for thermal therapy has limitations when quantitatively tracking temperature or temperature changes. This is because different tissues have different activation energies, which imply different temperature responses for different tissues. In addition, higher temperatures $\left(>40^{\circ} \mathrm{C}\right)$ cause irreversible changes in the tissues thereby confounding the temperature data.

\subsection{Proton Resonance Frequency Shift (PRFS)}

For a given proton, the resonance frequency is determined by the local magnetic field. In the case of water two protons share electrons with the oxygen atom. The electron has a large magnetic moment $\left(\sim 2000 \times m_{p}\right)$. In addition, the orbiting electrons will react to the external $\bar{B}_{o}$ field and shield the proton such that the field at the location of the proton is reduced. The screening effect in the water molecule is spatially average due to thermal motion of the molecule. Therefore, the water resonance frequency is temperature dependent. The temperature coefficient has been measured to be: $\mathrm{k} \sim 0.01 \mathrm{ppm}$ (partsper-million). For example, the resonance frequency of water in a 1.5 Tesla field is $\sim 64 \times 10^{6} \mathrm{~Hz}$. Therefore, at this field strength, the frequency will change by $\sim 0.64 \mathrm{~Hz} /{ }^{\circ} \mathrm{K}$. It may seem that a measurement of such a small frequency change is not practical. However, the MRI scanner is ideally suited to make phase measurements with high precision. The quadrature detection system used to demodulate the RF signal is a high-precision, phase-sensitive detector, limited only by the accuracy of the RF source and signal noise. Therefore, small changes in the resonance signal will result in phase offsets in the phase data, $\phi(x, y)$ of the reconstructed complex MR image (see section 2). This technique was proposed and implemented by De Poorter ${ }^{18}$ and Ishihara ${ }^{19}$. Measuring the phase offsets requires no special pulse sequences or hardware. Typically a gradient echo sequence is used (Figl1).

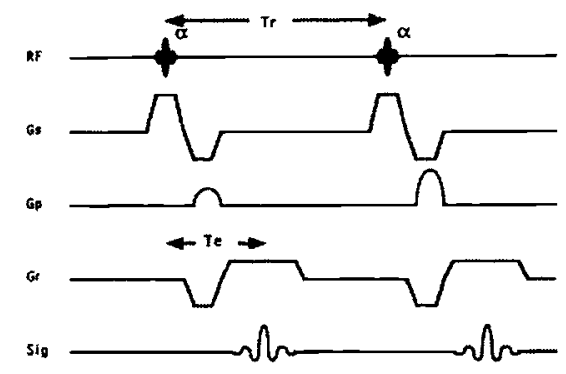

Fig. 11: Gradient echo pulse sequence. Typical image parameters are: $\mathrm{Tr}=34 \mathrm{~ms}, \mathrm{Te}=20 \mathrm{~ms}, \mathrm{FOV}=160 \mathrm{~mm}$, slice $=4 \mathrm{~mm}, \mathrm{BW}=+/-16 \mathrm{kHz}, 256 \times 256$ matrix,Nex=1

Temperature change information is accumulated in the phase term of the reconstructed real and imaginary images. In practice, the measurement is made by first acquiring a baseline image of the object that has some equilibrium temperature distribution. The baseline phase data, $\phi(x, y)_{o}$, are subtracted from phase data, $\phi(x, y)_{n}$, acquired in subsequent images having differing temperature distributions. The phase difference can be related to temperature by: 


$$
\Delta \phi(x, y)_{n}=2 \pi k \omega_{o} T_{E} \Delta T \quad \text { eq } 20
$$

The phase difference is proportional to the echo time, $T_{E}$, since the phase difference accumulates linearly with time. The pulse sequence parameters can be tailored to the particular application. The optimization of the gradient echo pulse sequence for thermal ablation using high intensity US was reported by $\mathrm{Chung}^{20}$.

The PRFS technique has been shown in vitro to have a sensitivity of $0.007-0.009 \mathrm{ppm}$, and a linear relation with temperature in tissue up to $50{ }^{\circ} \mathrm{C}^{21}$. The temperature sensitivity in a water-based gel phantom was measured by McFall et.al ${ }^{22}$ to be $0.0095 \mathrm{ppm}$ with resolution of $\sim 0.2^{\circ} \mathrm{C}$ in region of interest (ROI) volumes of $0.5 \mathrm{~cm}^{3}$. In the same report, the in vivo sensitivity of canine tissues (muscle, brain and tumor (sarcoma)) was found to be $\sim 0.007 \pm .0005 \mathrm{ppm}$, which is consistent with the in vitro results. The in vivo temperature and spatial resolution achieved was $\sim 0.6^{\circ} \mathrm{C}$ in $0.16 \mathrm{~cm}^{3}$.

The above in vivo results were obtained with temperature exposures less than $50^{\circ} \mathrm{C}$ that lasted for time periods of an hour or more using a RF phase array heating device. The temperature tracking was reversible in this range(see Fig 12).

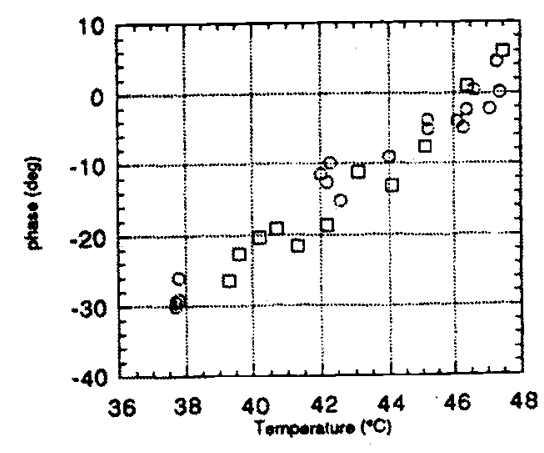

Fig. 12: PRFS vs. Temperature in canine sarcoma. Heating data are circles; cooling data are squares.

Similar data have been obtained using focused US heating. Hynynen et.al ${ }^{7}$ used the PRFS technique to measure in vivo temperatures in the focal zone of $1.5 \mathrm{MHz}$ US transducer. They were able to track the temperature evolution in time. Tissue damage was correlated US intensity and temperature.

Another recent $i v$ vivo application was reported by Smith ${ }^{23}$. This work used the PRFS technique to mapped temperature distributions associated with an intracavity US array. This device was designed to heat the human prostate via the rectum. They concluded that this technique should be feasible for human procedures.

Carter et. al reported a clinical investigation of the feasibility of using PRFS thermometry in human subjects ${ }^{24}$. Five patients having high-grade sarcoma in the lower extremity were administered hyperthermia while PRFS images were simultaneously acquired. 
Therapy was delivered with a RF phased array. Invasive temperature measurements were made with fiberoptic temperature probes. Correlation among the invasive probe measurements and PRFS phase changes were made using a linear regression model. These data yield a sensitivity factor of $\mathrm{k}=0.012 \mathrm{ppm}$, and temperature resolution of $\pm 0.9^{\circ} \mathrm{C}$ in a $1 \mathrm{~cm}^{3}$ voxel. The temperature images were better on some subjects and worse on others. In some cases the resolution was on the order of $0.5^{\circ} \mathrm{C}$ per $1 \mathrm{~cm}^{3}$ voxel. The variation can be associated with subject motion and MR system stability.

The PRFS technique is the method of choice for MR thermometry at this time. It works well in phantom and ex vivo applications. However, it has some important limitations that will impede some applications. Since the voxel phase assignment comes from the field gradients, Gy-x and Gy-y, object movement relative to the gradient fields will change $\phi(x, y)$. Therefore, gross motion invalidates the reference or baseline image. Small motions or tissue displacements can introduce large errors in the difference images. The net result is that the resolution in vivo is generally a factor of 3 times worse than that obtained ex vivo or in a phantom. Also, the stability of $\mathrm{B}_{\mathrm{o}}$ and associated shim currents produce significant phase drifts in the data. These problems are more severe for procedures that require measurements over extended time periods $(30-60 \mathrm{~min})$. The impact on procedures that involve rapid temperature elevations for short times is less sensitive to drift and motion problems.

\subsection{Apparent Diffusion Coefficient}

The free water molecules in tissue are in constant motion. The molecules are bound together by energy $\varepsilon_{a}$, but also have an average kinetic energy equal $k T$. The migration or diffusion of an individual molecule from one location to another is therefore governed by the Boltzman factor, $e^{\frac{-\varepsilon_{a}}{k T}}$, and the diffusion relation:

$$
\overline{\Delta r^{2}} \propto D t \quad \text { eq } 21
$$

where: $\overline{\Delta r^{2}}$ is the average displacement squared, $D=D_{o} e^{\frac{-\varepsilon_{a}}{k T}}$ is the diffusion coefficient, and $\mathrm{t}$ is the elapsed time from when the molecule was at $r=0$.

The activation energy for free water is $\sim 0.18 \mathrm{eV}$, therefore $\frac{d D}{d T}=0.024 \frac{\mathrm{mm}^{2}}{\sec ^{\circ} K}$.

It has been shown that the temperature dependence of the apparent diffusion coefficient (ADC) can be measured with MRI ${ }^{25,26}$.

Throughout an MR image acquisition sequence, water molecules are diffusing from one voxel to another. Therefore, there is some random mixing of the phase encoded protons due to this diffusion. However, since the encoding gradients are relatively weak, the intervoxel mixing of protons has a small attenuation effect on the resonance signal 
relative to a signal in which all the protons remain stationary within the voxels. Clearly this effect is proportional to strength, the duration of the gradient, and how rapidly the protons diffuse. The amount of diffusion mixing can be measured in a clever way by applying two large but short-duration gradients along some direction of interest. These gradients are of the same strength and duration but opposite in sign. They are also separated by time interval $\Delta t$. The effect of these gradients will cancel for protons that remain stationary in space since the phase advance produced by first gradient will be reversed by the second gradient. Their contribution to the signal will remain the same. Protons that have migrated to different locations along the direction of the applied gradient will have phase offsets proportional to the diffusion-distance traveled in the time $\Delta t$. These diffusing protons will be out of phase with the local stationary protons. Because the protons have an encoded large phase shift due to the large gradients, these protons will not add to the coherent refocused signal, and the signal received will be decreased relative to that obtained without additional diffusion gradients. Since this mixing is a random process proportional to gradient strength, duration, and the diffusion constant, the signal attenuation will be:

$$
\frac{S(b, D)}{S(b, D)_{o}}=e^{-b D}
$$

where:

$$
b=\gamma^{2} \int_{0}^{T E}\left[\int_{0}^{t} G\left(t^{\prime}\right) d t\right]^{2} d t
$$

For a spin echo signal acquisition (see Fig-13): $b=(\gamma G \delta)^{2}(\Delta-3 \delta)^{27}$

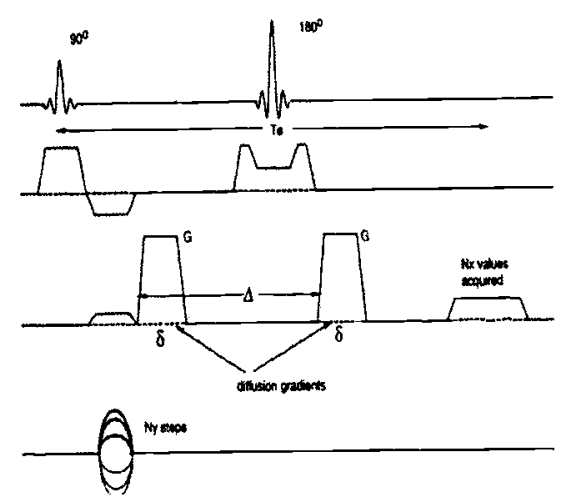

Fig. 13: Spin-echo sequence with diffusion gradients symmetrically applied before and after the $180^{\circ} \mathrm{RF}$ pulse

The use of diffusion imaging for non-invasive thermometry was proposed by Le Bihan in $1989^{26}$. Delannoy et.al ${ }^{28}$ demonstrated that diffusion imaging could used to measure temperature in a water-based gel phantom with a temperature resolution of $\sim 0.5^{\circ} \mathrm{C}$. Additionally, they accomplished this using a clinically relevant RF phased array heating device. The heating was gated so that the RF power was off during image acquisitions. In a highly controlled phantom experiment, Zhang et. al. ${ }^{29}$ demonstrated that diffusion thermometry could give temperature resolution of $0.2^{\circ} \mathrm{C}$ in. $0.3 \mathrm{~cm}^{3}$ voxels. 
Unfortunately, in vivo results were not as impressive. Diffusion imaging is exquisitely sensitive to object motion during the image acquisition. This is not a surprise since the objective is to measure the effects of molecular motion. Therefore a standard spin echo pulse sequence such as that in Fig.13 is completely inadequate for in vivo applications.

Experiments in vivo using a single shot echo-planar imaging technique (see Fig.13) were reported by McFall et. al. ${ }^{30}$.

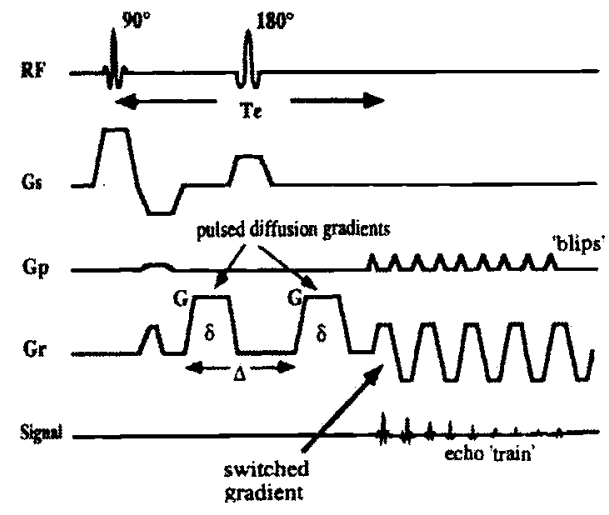

Fig. 14: The echo-planar pulse sequence captures all of $\mathrm{k}$-space in a single acquisition by using switching gradients with phase encoding "blips" between echoes.

These experiments were performed in the canine brain of normal animals. The animals were anaestitzed though out the heating procedure. A RF phased array was used to induce whole body hyperthermia. The imaging was cardiac gated to minimize pulsitile motion in the brain during the image acquisition. As whole body temperature approached $42^{\circ} \mathrm{C}$, panting occurred and the dogs were paralyzed with atracurium to control breathing motion as well. Under these conditions, temperature resolution of $0.9^{\circ} \mathrm{C}$ in $0.89 \mathrm{~cm}^{3}$ was achieved. The measured sensitivity was $0.02 \frac{\mathrm{mm}^{2}}{\mathrm{sec}^{\circ} \mathrm{C}}$ with $\varepsilon_{a}=0.23 \mathrm{eV}$.

More recently, improvements in hardware and software have produced higher and faster gradient fields and reductions in susceptibility artifacts. Thus, echo planar imaging has significantly improved as well. Using fast high-field gradients, MacFall et. al. examined the effect of tissue anisotropy on the temperature sensitivity of $(A D C)^{31}$. This was done in vivo in the canine brain. The results show that although the ADC varies in the anisotropic white matter, the sensitivity to temperature remained the same $\left(\sim 2.5 \% /^{\circ} \mathrm{C}\right)$. To date there has not been a report of using the ADC technique in a clinical therapy application.

\section{Summary}

We have reviewed the feasibility for using MRI as a means for non-invasively accessing temperature in thermal therapies. Four temperature sensitive parameters have been found useful for this application. The $T_{1}, T_{2}$ relaxation times are temperature dependent. However, they both suffer from irreversible effects with regard to tracking temperature above certain temperature thresholds. This does not a totally negate the use of these 
relaxation parameters because these irreversible effects provide image contrast indicative of tissue damage. This is extremely useful for treatment assessment.

The diffusion weighted technique has a high temperature sensitivity coefficient of $\sim 2.5 \%{ }^{\circ} \mathrm{C}$. However, this sensitivity does not translate into higher resolution temperature measurements in vivo because of motion effects. Rapid imaging is necessary for minimizing motion artifact with the diffusion technique. This requires large gradient fields and fast rise times that often produce susceptibility artifacts and geometric distortion in the diffusion-weighted images. Most malignant tissues have long $T_{2}$ relaxation times and this is advantageous for diffusion imaging. Unfortunately, normal tissue like muscle with a short $T_{2}$ is poorly imaged by this technique. This reduces the usefulness of the technique for evaluation of temperature in these normal tissues.

The PRFS temperature imaging has very good sensitivity as well as high spatial resolution. It appears at this time that this MR temperature measurement technique is the most robust. DePoorter ${ }^{18}$ compared PRFS thermometry with diffusion weighted thermometry and concluded that the PRFS was better with regard to acquisition time and temperature resolution. Recently Wlodarczyk et al. ${ }^{32}$ systematically compared four techniques, $T_{1}$, diffusion, PRFS and a contrast agent that had a large temperature dependent resonance frequency shift. They also concluded that the water PRFS is the better technique.

One might question the need for quantitative non-invasive measurements of temperature for thermal therapy, particularly with regard to ablative therapies. It may well be possible, for minimally invasive procedures, to incorporate sensors in heating devices such that invasively measured temperatures together with thermal modeling will be sufficient for safe and effective delivery of therapy. However, for non-invasive applications, non-invasive temperature measurements will be necessary. Also, for successful therapy, the measurements will have to be adequate to quantify the thermal dose delivered. Therefore, MRI will play a large role in the future of thermal therapeutic applications.

\section{ACKNOWLEDGMENTS}

This work supported in part by NIH/NCI grant \#5PO1 CA42745-13.

\section{REFERENCES}

1. B. Knuttel, H.P. Juretschke, "Temperature measurements by nuclear magnetic resonance and its possible use as a means of in vivo noninvasive temperature measurement and for hyperthermia treatment assessment," Recent Results in Cancer Research 101, pp. 109-118, 1986.

2. F.A. Jolesz, A.R. Bleier, P. Jakab, P.W. Ruenzel, K. Huttl, G.J. Jako, "MR imaging of laser-tissue interactions," Radiology 168, p. 249-253, 1988.

3. R. Matsumoto, K. Oshio, F.A. Jolesz, "Monitoring of laser and freezing-induced ablation in the liver with T1-weighted MR imaging," JRMI 2, pp. 555-562, 1992.

4. K. Hynynen, A. Darkazanli, E. Unger, J.F. Schenck, "MRI-guided noninvasive ultrasound surgery," Med. Phys. 20(1), pp. 107-115, 1993. 
5. C. Damianou, K. Hynynen, "The effect of various physical parameters on the size and shape of necrosed tissue volume during ultrasound surgery," J. Acoust. Soc. Am. 95(3), pp. 1641-1649, 1994.

6. K. Hynynen, A.H. Dhung, V. Colucci, F.A. Jolesz, "Potential adverse effects of high-intensity focused ultrasound exposure on blood vessels in vivo," Ultrasound in Med. \& Biol. 22(2), pp. 193-201, 1996.

7. K. Hynynen, N.I. Vykhodtseva, A.H. Chung, V. Sorrentino, V. Colucci, F.A. Jolesz, "Thermal effects of focused ultrasound on the brain: Determination with MR imaging," Radiology 204, 247-253, 1997.

8. C.J. Eskey, A.P. Koretsky, M.M. Domach, R.K. Jain, "2H-nuclear magnetic resonance imaging of tumor blood flow: Spatial and temporal heterogeneity in a tissue-isolated mammary adenocarcinoma," Cancer Research 52, p. 6010-6019, 1992.

9. C.A. Belfi, C.R. Paul, S. Shan, F.Q. Ngo, 1994, "Comparison of the effects of hydralazine on tumor and normal tissue blood perfusion by MRI," Int. J. Rad. Onc., Biol., Phys. 29(3), pp. 473-490, 1994.

10. C.A. Belfi, L.L. Ting, S.J. Hassenbusch, M. Tefft, F.Q. Ngo, "Determination of Changes in Tumor Blood Perfusion after Hydralazine Treatment by Dynamic Paramagnetic-Enhanced Magnetic Resonance Imaging," Int. J. Rad. Onc., Biol. Phys. 22(3), pp. 477-482, 1992.

11. M.W. Dewhirst, H.D. sostman, K.A. Leopold, H.C. Charles, D. Moore, R.A. Burn, J.A. Tucker, J. Harreson, J.R. Oleson, "Soft-tissue sarcomas: MR imaging and MR spectroscopy for prognosis and therapy monitoring - Work in progress," Radiology 174, pp. 847-853, 1990.

12. H.D. Sostman, D.M. Prescott, M.W. Dewhirst, R.K. Dodge, D.E. Thrall, R.L. Page, J.A. Tucker, J. Harrelson, K.A. Leopold, J.R. Oleson, H.C. Charles, "MRI/MRS for prognostic evalutaion in soft tissue sarcomas," Radiology 190, pp. 269-275, 1994.

13. D.M. Prescott, H.C. Charles, H.D. R.K. Dodge, D.E. Thrall, R.L. Page, J.A. Tucker, J.M. Harrelson, K.A. Leopold, J.R. Oleson, M.W. Dewhirst, "Therapy monitoring in human and canine soft tissue sarcomas using magnetic resonance iamging and spectroscopy," Int. J. Rad. Onc., Biol. Phys 28, pp. 415-423, 1994.

14. C.J. Lewa, Z Majewska, "Temperature relationships of proton spin-lattice relaxation time T1 in biological tissues," Bulletin Du Cancer 67(5), pp. 525-530, 1980.

15. D.L. Parker, V. Smith, P. Sheldon, L.E. Crooks, W. Fussell, "Temperature distribution measurements in two-dimensional NMR imaging," Med. Phys. 10(3), pp. 321-325, 1983.

16. R.J. Dicksinson, A.S. Hall, A.J. Hind, I.R. Young, "Measurement of change in tissue temperature using MR imaging," J. Computer Assisted Tomography 10(3), pp. 468-472, 1986.

17. H.E. Cline, J. Hynynen, C.J. Hardy, R.D. Watkins, J.F. Schenck, F.A. Jolesz, "MR temperature mapping of focused ultrasound surgery," Magnetic Resonance in Medicine 31, pp. 628-636, 1994.

18. J. De Poorter, C. De Wagter, Y. De Deene, C. Thomsen, R. Stahlberg, E. Achten, "The proton-resonance-frequency-shift method compared with molecular diffusion for quantitative measurement of two-dimensional time-dependent temperature distribution in a phantom," J. Magn. Res. 103, pp. 234-241, 1994. 
19. Y. Ishihara, A. Calderon, H. Watanabe, K. Okamoto, Y. Suzuki, K. Kuroda, Y.Suzuki,"Aprecise and fast temperature mapping using water proton chemical shift," Mag Res Med34, 814-823, 1995.

20. A.H. Chung, K. Hynynen, V. Colucci, K. Oshio, H.E. Cline, F.A. Jolesz, "Optimization of spoiled gradient-echo phase imaging for in vivo localization of a focused ultrasound beam," Magnetic Resonance in Medicine 36, pp. 745-752, 1996.

21. K. Kuroda, K. Abe, S. Tsutsumi, Y. Ishihara, Y. Suzuki, K. Sato, "Water proton magnetic resonance spectroscopic imaging," Biomed. Thermol. 13, pp. 43-62, 1993.

22. J.R. MacFal, D.M. Prescott, H.C. Charles, T.V. Samulski, " ${ }^{1}$ H MRI phase thermometry in vivo in canine brain, muscle and tumor tissue," Medical Physics 23(10), pp. 1775-1782, 1996.

23. N.B. Smith, M.T. Buchanan, K. Hynynen K., "Transrectal ultrasound applicator for prostate heating monitored using MRI thermometry," Int. J. Rad. Onc., Biol. Phys 43(1), pp. 217-225, 1999.

24. D.L. Carter, J.R. MacFall, S.T. Clegg, X. Wan, D.M. Prescott, H.C. Charles, T.V. Samulski, "Magnetic resonance thermometry during hyperthermia for human high-grade sarcoma," Int. J. Radiat. Oncol. Biol. Phys. 40(4), pp. 815$822,1998$.

25. D. LeBihan, E. Breton, D. Lallemand, M.L. Aubin, J. Vignaud, M. Laval-Jeantet, "Separation of diffusion and perfusion in intravoxel incoherent motion MR imaging," Radiology 168, 1988.

26. D. LeBihan, J. Delannoy, R.L. Levin, "Temperature mapping with MR imaging of molecular diffusion: Application to hyperthermia," Radiology 171, pp. 853$857,1989$.

27. E.O. Stejskal, J.E. Tanner, "Spin diffusion measurements: spin echoes in the pressence of a time-dependent field gradient," J. Chem. Phys. 42, pp 288, 1965.

28. J. Delannoy, D. LeBihan, D.I. Hoult, R.L. Levin, "Hyperthermia system combined with a magnetic resonance imaging unit," Med. Phys. 17(5), pp. 855-860, 1990.

29. Y. Zhang, T. Samulski, W. Joines, J. Maltiello, R. Levin, D. LeBihan, "On the accuracy of noninvasive thermometry using molecular diffusion magnetic resonance imaging," Int. J. Hyperthermia 8, pp. 263-274, 1992.

30. J. MacFall, D. Prescott, E. Fullar, T. Samulski, "Temperature dependence of canine brain tissue diffusion coefficient measured in vivo with magnetic resonance echo-planar imaging," Int. J. Hyperthermia 11, pp. 73-86, 1995.

31. J.R. MacFall, T.V. Samulski, D.M. Prescott, J. Poulsen, "Temperature dependence of the apparent diffusion coefficent in anisotropic canine brain tissue invivo for different diffusion directions. Presented at the 5th Meeting of the Soceity of Magnetic Resonance, San Francisco, CA, April 12-18, 1997; 1:1699.

32. W. Wlodarczyk, M. Hentschel, P. Wust, R. Noeske, N. Hosten, H. Rinneberg, R. Felix, "Comparison of four magnetic resonance methods for mapping small temperature changes," Phys. in Med. \& Biol. 44(2), pp. 607-624, 1999. 